\title{
Water oxidation at hematite photoelectrodes: the role of surface states
}

\section{Benjamin Klahr $^{+\dagger}$, Sixto Gimenez ${ }^{+\ddagger}$, Francisco Fabregat-Santiago , Thomas Hamann* ${ }^{\star}$, Juan Bisquert* ${ }^{\star \star}$}

¥Photovoltaics and Optoelectronic Devices Group, Departament de Física, Universitat Jaume I, 12071 Castelló, Spain

${ }^{\dagger}$ Department of Chemistry, Michigan State University, East Lansing, MI 48824-1322

Email:bisquert@fca.uji.es

hamann@chemistry.msu.edu

${ }^{+}$both authors contributed equally to this work

13 June 2013

Published as

Klahr, B.; Gimenez, S.; Fabregat-Santiago, F.; Hamann, T.; Bisquert, J. Water Oxidation at Hematite Photoelectrodes: The Role of Surface States, Journal of the American Chemical Society 2012, 134, 4294-4302. Doi: $10.1021 / \mathrm{ja} 210755 \mathrm{~h}$ 


\section{Abstract}

Hematite $\left(\alpha-\mathrm{Fe}_{2} \mathrm{O}_{3}\right)$ constitutes one of the most promising semiconductor materials for the conversion of sunlight into chemical fuels by water splitting. Its inherent drawbacks related to the long penetration depth of light and poor charge carrier conductivity are being progressively overcome by employing nanostructuring strategies and improved catalysts. However, the physical-chemical mechanisms responsible for the photoelectrochemical performance of this material ( $J(V)$ response) are still poorly understood. In the present study we prepared thin film hematite electrodes by Atomic Layer Deposition to study the photoelectrochemical properties of this material under water splitting conditions. We employed Impedance Spectroscopy to determine the main steps involved in photocurrent production at different conditions of voltage, light intensity and electrolyte $\mathrm{pH}$. A general physical model is proposed, which includes the existence of a surface state at the semiconductor/liquid interface where holes accumulate. The strong correlation between the charging of this state with the charge transfer resistance and the photocurrent onset provides new evidence of the accumulation of holes in surface states at the semiconductor/electrolyte interface, which are responsible for water oxidation. The charging of this surface state under illumination is also related to the shift of the measured flat band potential. These findings demonstrate the utility of Impedance Spectroscopy in investigations of hematite electrodes to provide key parameters of photoelectrodes with a relatively simple measurement. 


\section{Introduction}

As part of the quest to develop better and cleaner energy conversion and storage systems, the direct conversion of sunlight into chemical fuels has become a subject of renewed interest. One attractive example is the use of semiconductors to harness solar photons to split water, thereby producing hydrogen as a chemical fuel. In order to achieve this, a given material must satisfy a number of stringent requirements including visible light absorption, efficient charge carrier separation and transport, facile interfacial charge-transfer kinetics, appropriate positions of the conduction and valence band energy levels with respect to required reaction potentials and good stability in contact with aqueous solutions 1 While such systems were heavily investigated several decades ago, no material so far has fulfilled all the required conditions R $_{3}$ Recent advances in nanotechnology and catalysis, however, greatly increase the prospects of developing a combination of materials capable of efficient conversion of sunlight to chemical fuels 4

Hematite $\left(\alpha-\mathrm{Fe}_{2} \mathrm{O}_{3}\right)$ is a very promising material for photoelectrochemical (PEC) water splitting due to its combination of sufficiently broad visible light absorption, up to $590 \mathrm{~nm}$, and excellent stability under caustic operating conditions However, hematite electrodes are adversely affected by a number of factors including a long penetration depth of visible light due to its indirect band gap transition and a very short minority carrier lifetime and mobility; this combination hinders efficient collection of the minority carriers via the required interfacial charge-transfer reactions. Considerable effort has been devoted to improving the actual efficiency by employing nanostructuring strategies, which disconnects the light absorption and charge collection processes, as well as introducing dopant atoms into the lattice surface for the water oxidation (oxygen evolution) reaction seems to be sluggish, which allows for increased recombination with a concomitant loss of photocurrent and efficiency. A detailed understanding of the water oxidation reaction at the hematite electrode surface is therefore very important in devising strategies to overcome this kinetic barrier. Recently, a series of studies based on different characterization techniques have improved our understanding of the factors controlling the water splitting performance of hematite photoelectrodes $9-12$ These works have pointed out the crucial role of the accumulation of holes at the surface of hematite electrodes under visible light irradiation in decreasing the photocurrent; however a clear discrimination of recombination and charge transfer rates, as well as the role of the applied voltage, has not yet been elucidated. 
In this work we prepared a series of thin film hematite electrodes via atomic layer deposition (ALD) to investigate the PEC properties of this material under water splitting conditions. ALD allows the reproducible deposition of pinhole-free, conformal films with a controllable thickness with angstrom resolution ${ }^{13-15}$ Importantly, the self-limiting gas phase mechanism of ALD is an ideal technique for depositing thin films on high aspect ratio scaffolds which are deposited identically to the thin films used herein 16 Thus, the lessons learned on the model thin films can be directly applied to nanostructured electrodes prepared by ALD. The thin, planar films used herein provide an ideal electrode for fundamental studies as they avoid the complexity and irregularities of most nanostructured surfaces. We employed Impedance Spectroscopy (IS) to determine the main steps involved in photocurrent production under different conditions: the controlled variation of voltage, light intensity and electrolyte $\mathrm{pH}$. Our results are based on the analysis of surface state capacitance $17-20$ a concept developed in the 1980s, which provides new evidence of the accumulation of holes in surface states at the semiconductor/electrolyte interface. We have found that the chargetransfer reaction to oxidize water occurs from these surface states rather than from holes from the valence band; the photocurrent onset appears only after holes start to accumulate in these surface sites. These findings further establish IS as an excellent tool to investigate different configurations of hematite electrodes in order to determine the charge dynamics with a relatively simple measurement.

\section{Background}

Impedance Spectroscopy is a well established method in PEC that uses a small voltage-tocurrent ratio, the impedance $Z$, measured at different angular frequencies, $\omega$, at a given condition of steady state. The spectroscopic scan over the relevant window of frequencies is resolved into a combination of resistances and capacitors in a given arrangement, called the equivalent circuit (EC). The EC is a useful tool for the interpretation of experimental results, provided that the different elements and their particular arrangement in a network possess physical meaning. Additionally, the EC describes the sequential displacement/accumulation of carriers in specific processes that compose the system, together with the specific places that produce steps of Fermi levels of the carriers, in relation to the electrostatic potential distribution in the system. It is sometimes claimed that the interpretation of the results based on EC is ambiguous, based on the fact that different equivalent representations of a given $Z(\omega)$ function are possible. While there is a variety of possible representations of a single physical-chemical model, it is also true that the EC allows for the conveyance of a great 
amount of information, especially when application of a simplified model is needed, according to the real amount of information contained in the data (in opposition to that contained in a general model), to avoid over-parameterization. Thus, while IS is straightforward to measure, the main problem is the extraction of information contained in the data, and this is greatly aided by using a sound EC, especially in a field where such methods have been tested over many years and a great deal of experience is available. It is important, therefore, to clarify the basis for a given approach to treat the experimental results, and we describe ours in the following.

We should keep in mind that the main goal for understanding the PEC operation of a photoelectrode is to explain the characteristic $J(V)$ behavior; that is, the current density with respect to applied bias voltage under steady state conditions. Indeed, the $J(V)$ characteristics are used to derive the efficiency, considering the number of photons impinging on the semiconductor 6 In IS, the small perturbation procedure provides the following quantity

$$
R_{\text {tot }}=\left(A_{s} \frac{\partial J}{\partial V}\right)^{-1}
$$

where $R_{t o t}$ is a total resistance, $R_{\text {tot }}=Z(\omega=0)$, and $A_{S}$ is the surface area of the electrode. Equation 1 shows the close relationship between $J(V)$ and impedance results, and how the latter is used to understand the former. The point is that with IS we can resolve the different components of $R_{t o t}$, while this is more difficult working directly with $J(V)$. As an example, suppose that the traffic of carriers from the back contact to the solution consists of two serial processes $R_{t o t}=R_{1}+R_{c t}$, one for transport and another one for interfacial charge transfer. The feature that enables us to separate the two added resistances is the capacitance. Considering the classical Randles circuit, this capacitance is associated with the Helmholtz layer at the electrode/solution interface, $C_{H}$, and affects only $R_{c t}$ in parallel.

For the interpretation of PEC measurements of illuminated and dark hematite electrodes we adopt a classical view that is depicted in Figure 1(a) this allows us to move directly to the discussion of the elements of EC to interpret the IS measurements, while we note that analytical formulations of this problem are well-known and have been amply discussed in the literature $22-25$ The model we suggest is shown in Figure 1(b) and highlights the central role of a surface state acting as a recombination center, trapping electrons from the conduction band

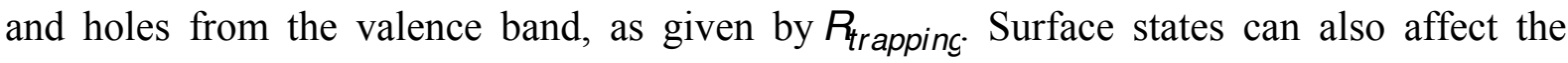
charge transfer of holes to the donor species in solution, as described by $R_{c t, t r a p}$, although another pathway for direct charge transfer from the semiconductor bands is included, $R_{c t, b u l k}$. A formal derivation of the interfacial impedance is given in Ref. 26. This EC can also be 
traced back to a large body of literature on IS of PEC system $\$ 18-207$ and can be considered standard knowledge, although a complete interpretation of the measured data is often elusive due to the complexity of this system. Therefore, some clarification of fundamental points seems necessary. As mentioned before, the crucial element for the analysis of resistances in IS are the capacitances and their combination in the EC, and we now discuss the capacitive elements in the EC. For a sufficiently thick (with respect to Debye screening length) and doped semiconductor material, there are two capacitances well established in the literature. First, the depletion layer capacitance, $C_{S C}$, described by the Mott-Schottky (MS) equation:

$$
\left(\frac{A_{S}}{C_{s c}}\right)^{2}=\frac{2}{q \kappa \varepsilon_{0} N_{D}}\left(V-E_{f b}-\frac{k_{B} T}{q}\right)
$$

where $V$ is the applied voltage, $E_{f b}$ is the flat band potential, $N_{D}$ is the dopant density, $k_{B}$ is Boltzmann's constant, $T$ the absolute temperature, $q$ is elementary charge, $\kappa$ is the dielectric constant of the semiconductor (taken to be 32 for the hematite) ${ }^{28}$ and $\varepsilon_{0}$ is the vacuum permittivity $\left(8.854 \times 10^{-12} \mathrm{C} \mathrm{V}^{-1} \mathrm{~m}^{-1}\right)$. In addition, there is the series connection of $C_{H}$, where both elements have been lumped into $C_{\text {bulk }}$. These two capacitances are dielectric in origin. In contrast to this, there is a third capacitance in this system that is of the type of a

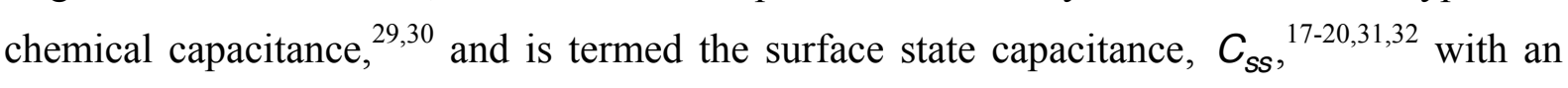
expression given by

$$
C_{s s}=A_{S} q N_{s s} \frac{\partial f_{s s}}{\partial E_{F n}}=A_{S} \frac{N_{s s} q^{2}}{k_{B} T} f_{s s}\left(1-f_{s s}\right)
$$

Here $N_{s s}$ is the surface density of the surface states, $f_{s s}$ the fractional occupancy of the state, and $E_{F n}$ the electron Fermi level of the state. 

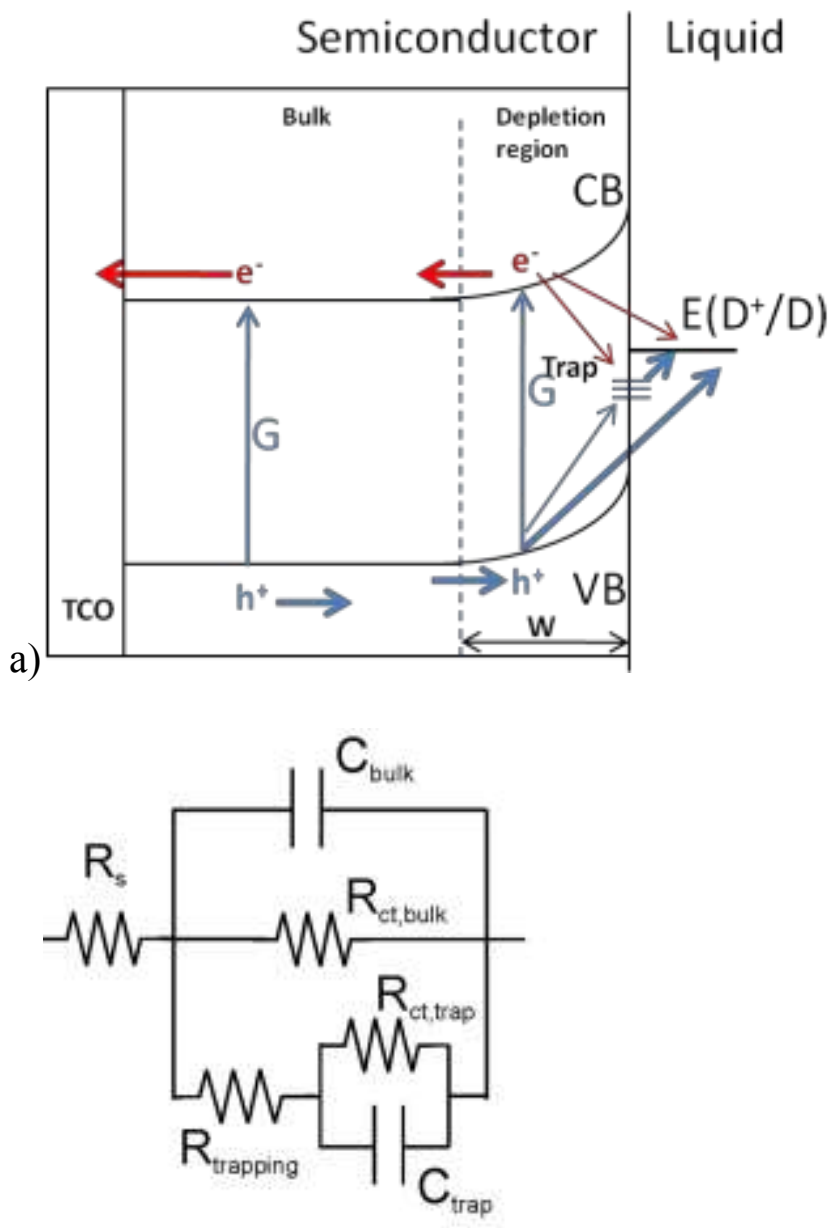

b)

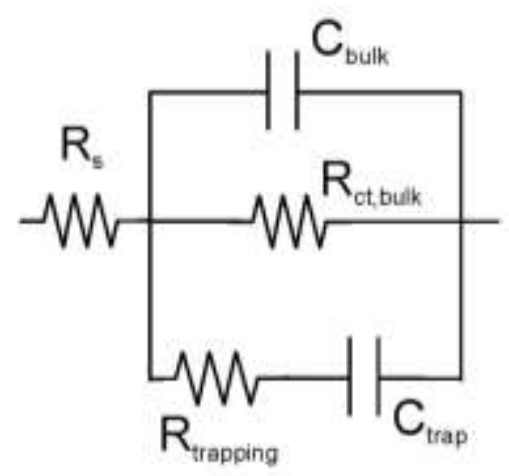

c)

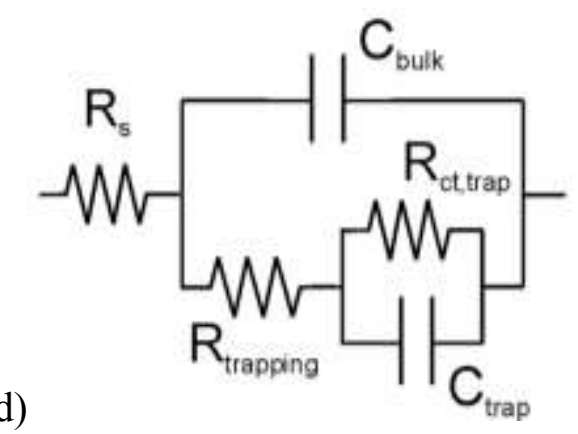

7 
e)

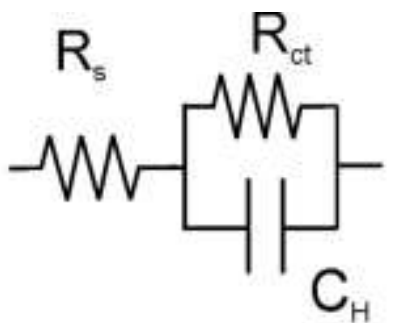

Figure 1. (a) Proposed physical model for the charge carriers dynamics in hematite electrodes, showing their generation, G, by light absorption, surface state trapping, and interfacial charge-transfer reactions. (b) Equivalent circuit corresponding to physical model in (a). (c) Simplified model used for IS interpretation created by removing $R_{c t, t r a p}$. (d) Simplified model used for IS interpretation created by removing $R_{c t, b u l k}$. (e) Randles circuit

According to Equation (3) the surface state capacitance traces a peak with respect to Fermi level variation at the point $E_{F n}=E_{s S}$, where $E_{s s}$ is the energy level of the surface state. Therefore, a voltage scan of the capacitance at intermediate frequencies (to remove the effect of $C_{\text {bulk }}$ ) allows a direct spectroscopic measurement of the surface states of a semiconductor in the energy axis. This method has been applied over many years, especially using an inert electrolyte that totally blocks the current flow $\left.1\right|_{\mid 2}$ However, in general we must be careful when establishing the relationship between the applied voltage and the energy of the surface state. We recall that in PEC system consisting of an n-type semiconductor in contact with a well defined redox couple, the voltage in a dark measurement relates to the difference of the Fermi level of electrons in the semiconductor, $E_{F n}$, and the redox potential of the redox couple, as indicated in Figure 2(a). When irradiated with suprabandgap illumination from the electrolyte side, electron-hole pairs are generated in the region where the light is absorbed, plus one diffusion length of the minority carriers ${ }^{21}{ }^{23}$ which are the holes in this case. Excess minority carriers are therefore created close to the interface, with the concomitant lowering of their own Fermi level, as indicated in Figure 2(b). The split of the Fermi levels produces a photovoltage, and for infinitely fast exchange of holes with the redox species across the interface, equilibrium would be achieved, where $E_{F p}^{\text {surface }}=E_{r e d o x}$. This means that the voltage will be given by $-q V=E_{F n}-E_{r e d o x}$, just as in Figure 2(a). The usual case, however, is that $E_{F p}^{\text {surface }}<E_{\text {redox }}$, due to sluggish exchange, particularly when intermediate steps for the reaction or surface states are involved ${ }^{34}$ Therefore, the Fermi level of holes at the surface becomes effectively disconnected from the applied voltage, and the problem requires a kinetic solution. 

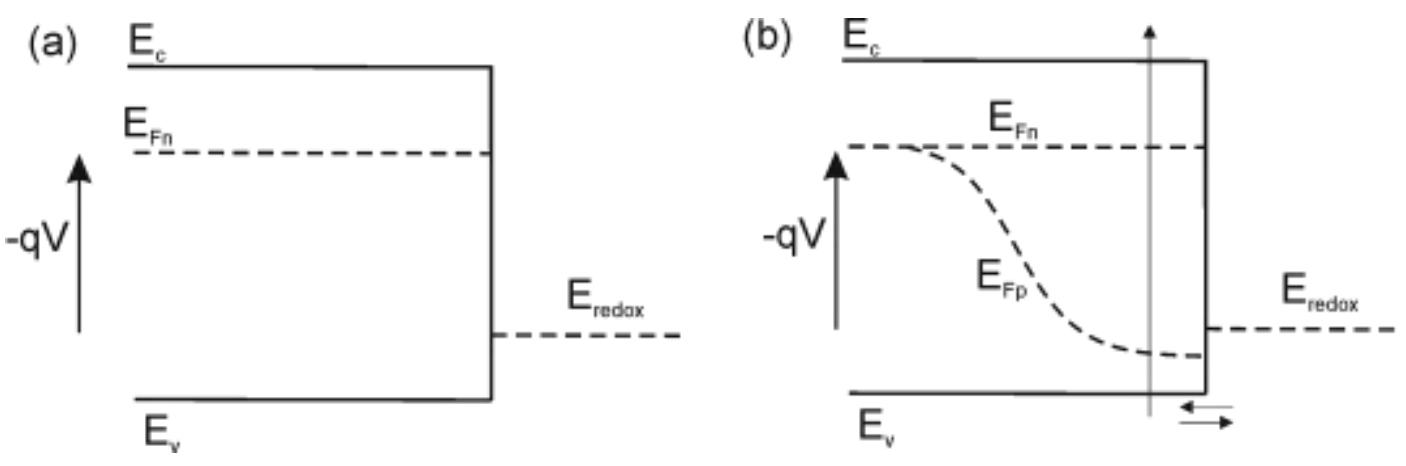

Figure 2. Scheme of a semiconductor with conduction band and valence band edges $E_{C}$ and $E_{V}$, and the quasi-Fermi levels of the respective states. (a) Applied voltage $V$ in the dark. (b) Photogeneration of electron hole pairs and charge transfer of hole to redox couple in solution.

One important point for the interpretation of the capacitance results is therefore the connection of the voltage with the occupation of the surface states, and the main consideration here is that $f_{s s}$ may be determined exclusively by kinetic factors 35 In Figure 3 we summarize the main cases for the interpretation of $C_{s S}$. Figure 3(a) shows the case of an electron trap that only exchanges electrons with the conduction band. In this case both the extended states and the trap are subjected to a unique Fermi level $E_{F n}$. This situation has been widely discussed in dye-sensitized solar cells in terms of the quasistatic approximation ${ }^{33 \beta^{7} 7}$ This case (a) is also valid in describing a surface state in the presence of a blocking electrolyte, so that the voltage really reads the position of the electron Fermi level in the surface state $\left.{ }^{1}\right|_{\mid 22}$ The second case, (b), is that of a surface state that transfers electrons to/from the solution. In this case, the Fermi level of the surface state can still be defined by kinetics, and is lower than the Fermi level of the free electrons, implying that the peak of the surface state appears at more negative voltage (more positive Fermi level position) than the real position of $E_{s s} 20$ A similar situation is found in Figure 3(c), where the trap states accept both electrons and holes and functions as a recombination center 39 The PEC situation relevant to this work, and also given in Figure 2, is shown in Figure 3(d). We see that the occupancy of the surface state is governed by the rates of trapping electrons and holes, and by the rate of charge transfer by the surface reaction with electrolyte species. It is obvious that there are different possible situations, depending on the different kinetic constants of these processes, which determine the position and size of the capacitance peak. A full description of the kinetic model requires analytical development beyond the scope of the present work. Another well known effect, is the shift of the energy levels of the semiconductor that is caused by surface charging. This effect is represented in Figure 3(e) for the case that the hole 
density in the surface state increases with respect to Figure 3(d). This effect produces a stabilization of the semiconductor capacitance, $C_{s c}$, due to the pinning of the Fermi level, and a displacement of the MS plots ${ }^{18-20}$ The displacement of MS is a function of the illumination, which changes the hole density in the surface state.
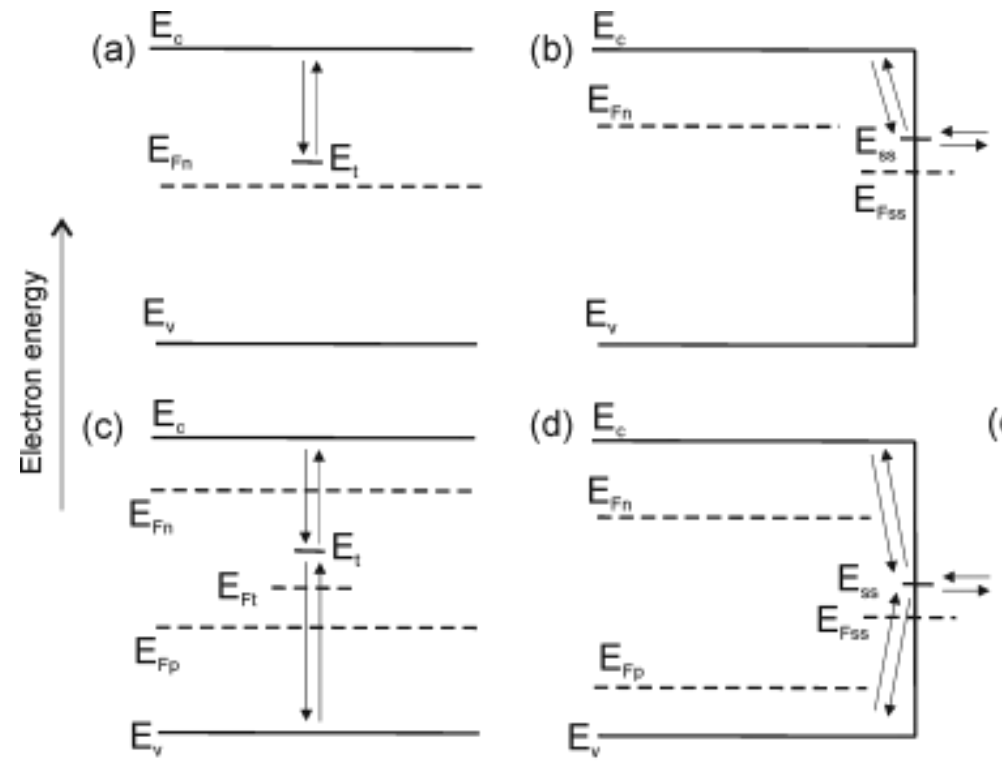

(d)

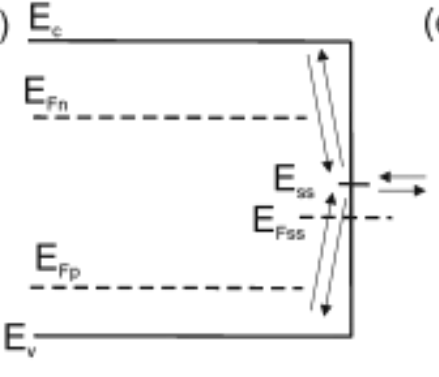

(e)

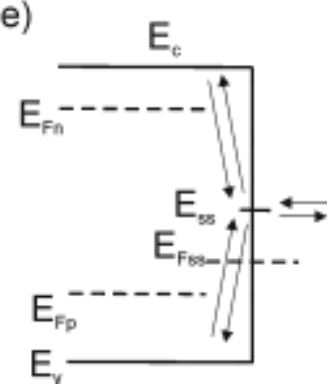

Figure 3. Scheme of a semiconductor with conduction band and valence band edges $E_{C}$ and $E_{v}$, a localized state in the bandgap $E_{t}$ or surface state $E_{s S}$, and the quasi-Fermi levels of the respective states. (a) Exchange of electrons only from conduction band. (b) Trapping and charge transfer of electrons. (c) Trapping of electrons and holes. (d) Trapping and charge transfer of electrons and holes and (e) displacement of bands by surface charging.

\section{Experimental}

Thin films of hematite were deposited on fluorine-doped tin oxide (FTO) coated glass substrates (Hartford Glass, $12 \Omega \mathrm{cm}^{-2}$ ) by atomic layer deposition (Savannah 100, Cambridge Nanotech Inc.) using a modified procedure to that described previously 40 The modification consisted of using both ozone and water as the oxidation source instead of just ozone, which results in increased growth rate and uniformity of the hematite films compared to those made using only ozone as the oxygen source ${ }^{41}$ A single precursor-oxidation cycle consisted of a 20 second ferrocene pulse followed by an oxidation sub-cycle which included 10 cycles of a $0.015 \mathrm{~s} \mathrm{H}_{2} \mathrm{O}$ pulse followed by a $6 \mathrm{~s}$ ozone pulse, where each sub-cycle was separated by a $5 \mathrm{~s}$ purge. This oxidation cycle is thus essentially a $60 \mathrm{~s}$ "wet" ozone pulse. All films in this experiment were prepared by 1,200 ALD cycles and measured to be $\sim 60 \mathrm{~nm}$ by absorption measurements (Perkin Elmer, Lambda 35 with a Labsphere integrating sphere) corrected for 
reflection as described previously, as well as ellipsometric measurements (Horiba Jobin Yvon, Smart-SE) 40

Electrolytes were prepared at pH 6.9 (employing a $0.1 \mathrm{M}$ phosphate buffer) and $\mathrm{pH} 13.3$ (0.1 M KOH). The $\mathrm{pH}$ was determined with Fisher Scientific Accumet $\mathrm{pH}$ meter. All aqueous solutions contained $200 \mathrm{mM} \mathrm{KCl}$ as a supporting electrolyte. Hematite electrodes were masked with a $60 \mu \mathrm{m}$ Surlyn film (Solaronix) with a $0.25 \mathrm{~cm}^{2}$ hole to define the active area and to prevent scratching of the thin films which were clamped to a custom made glass electrochemical cell. Surlyn films were adhered to the electrodes by heating to $120^{\circ} \mathrm{C}$. A homemade saturated $\mathrm{Ag} / \mathrm{AgCl}$ electrode was used as a reference electrode and high surface area platinum mesh was used as the counter electrode. Impedance spectroscopic and photoelectrochemical measurements were made with an Eco Chemie Autolab potentiostat coupled with Nova electrochemical software. IS data were gathered using a $10 \mathrm{mV}$ amplitude perturbation of between 10,000 and $0.01 \mathrm{~Hz}$. The IS data over this six order of magnitude variation of frequencies were simultaneously fit to the equivalent circuits described in the text using Zview software (Scribner Associates). The light source was a $450 \mathrm{~W}$ Xe arc lamp. An AM 1.5 solar filter and neutral density filters were used to simulate sunlight at $100 \mathrm{~mW} \mathrm{~cm}$; neutral density filters were also employed to reduce the light intensity to 33 and $10 \mathrm{~mW} \mathrm{~cm}$. All photoelectrochemical measurements were performed by shining light from the substrateelectrode (SE) interface. While all experiments shown in this work were performed multiple times, the data shown herein are from a single hematite electrode.

\section{Results and Discussion}

Plots of steady state photocurrent density, J, vs. applied voltage, $V$, curves in response to varying light intensities $-10,33$ and $100 \mathrm{~mW} \mathrm{~cm}^{-2}$ - are shown in Figure 4(a) for $\mathrm{pH} 6.9$ and Figure 4(b) for $\mathrm{pH}$ 13.3. Since the water oxidation potential and the hematite bands both shift at the Nernstian rate of $59 \mathrm{mV} / \mathrm{pH}$, the potentials were normalized to the real hydrogen electrode reference (RHE) ${ }^{42-44}$ The $J(V)$ curves under $100 \mathrm{~mW} \mathrm{~cm}^{-2}$ illumination (1 sun) are plotted vs RHE in Figure 4(c). The curves show remarkable overlap, however the performance of the electrodes at $\mathrm{pH} 13.3$ does show a somewhat improved current onset potential of about $100 \mathrm{mV}$. 


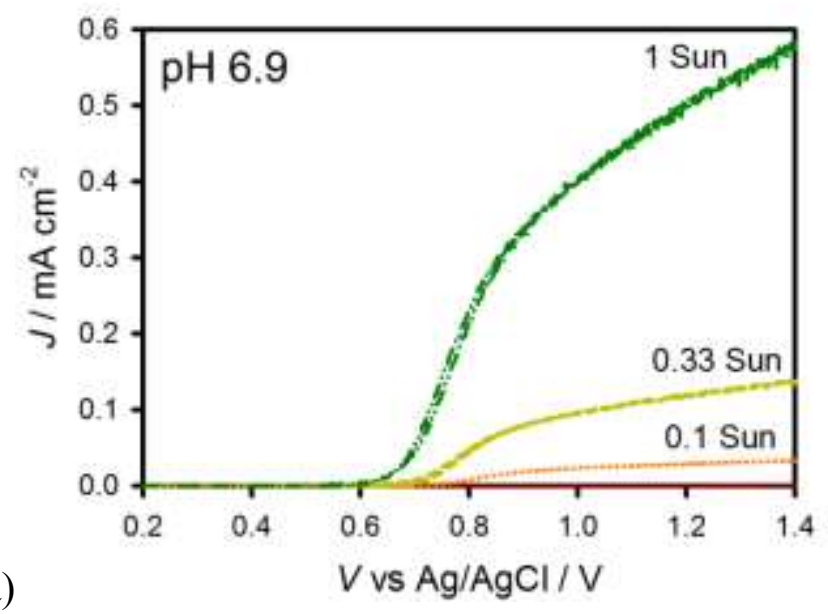

a)

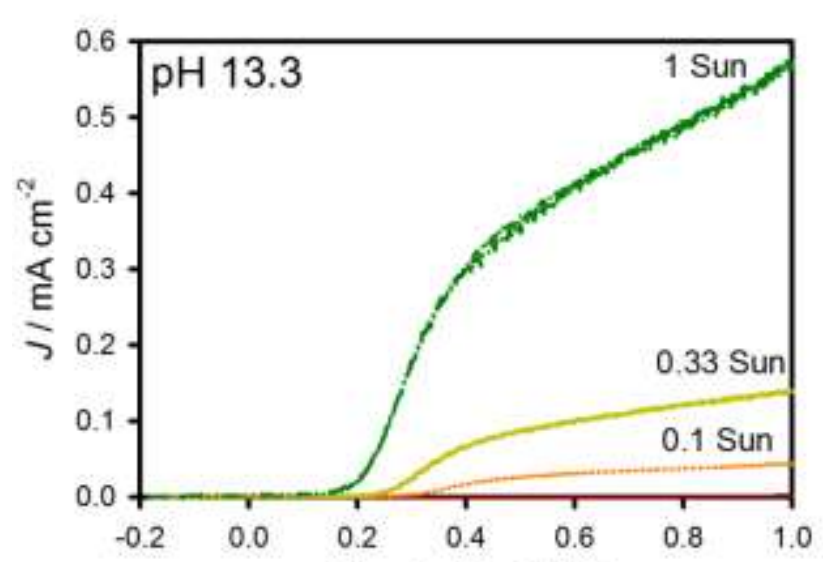

b)

$V$ vs Ag/AgCl/V

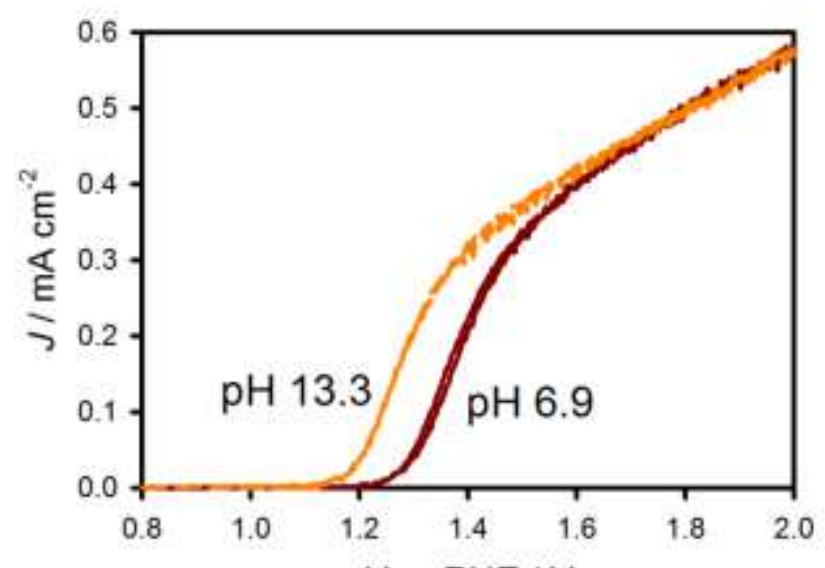

c)

$\checkmark$ vs RHE / V

Figure 4. $J(V)$ curves for a $60 \mathrm{~nm}$ thick $\mathrm{Fe}_{2} \mathrm{O}_{3}$ electrodes at $\mathrm{pH} 6.9$ (a) and 13.3 (b) under different illumination intensities; dark $J(V)$ curves are indistinguishable from the $\mathrm{x}$ axis on this scale. (c) $J(V)$ curves measured under 1 sun illumination for 2 different electrodes measured at $\mathrm{pH} 6.9$ and $\mathrm{pH} 13.3$ plotted on the RHE scale.

IS measurements were performed over the same potential range as the $J(V)$ curves, under each light intensity and in the dark, at $\mathrm{pH} 6.9$ and $\mathrm{pH}$ 13.3. Representative Nyquist plots 
under illumination are shown in Figure 5. Clearly there are two semicircles apparent in the impedance spectrum. Similar looking impedance spectra have recently been reported for hematite electrodes, and a variety of equivalent circuits put forth interpret these spectra ${ }^{11} .45$ In these analyses, the low frequency semicircle is generally attributed to the series arrangement of the depletion capacitance of the semiconductor $C_{S C}$ and the Helmholtz capacitance at the electrode surface, and the role of surface states has largely been ignored. The general EC proposed, which includes surface state hole trapping proposed in Figure 1(b), cannot unambiguously fit the impedance spectra since it does not discriminate between $R_{c t, b u l k}$ and $R_{c t, t r a p}$. Consequently, two simplifications of this general equivalent circuit have been employed, as shown in Figure 1(c) and 1(d). In these simplifications, either $R_{c t, \text { trap }}$ or $R_{c t, b u l k}$ is eliminated. The simplified equivalent circuits are excellent approximations if charge transfer (water oxidation) is dominated by one route, either from the valence band (Fig. 1(c)) or surface states (Fig. 1(d)). Both of the simplified models were used to fit the IS data under illumination and more consistent results were obtained for the model displayed in Figure 1(d) (See Supporting Information for a more detailed explanation, Fig. SI1). The simplified model in Figure 1(d) is therefore employed to derive the fit parameters from all the IS measurements under illumination for the analysis presented below. We note, however, that only one semicircle was observed for IS measurements in the dark at all applied potentials. In this case, since a second capacitance cannot be determined, a Randles circuit was employed to fit the data, Figure 1(e) which is equivalent to the simplified model in fig 1(b) without the $R_{\text {trapping }}$ and $C_{\text {trap }}$ elements.

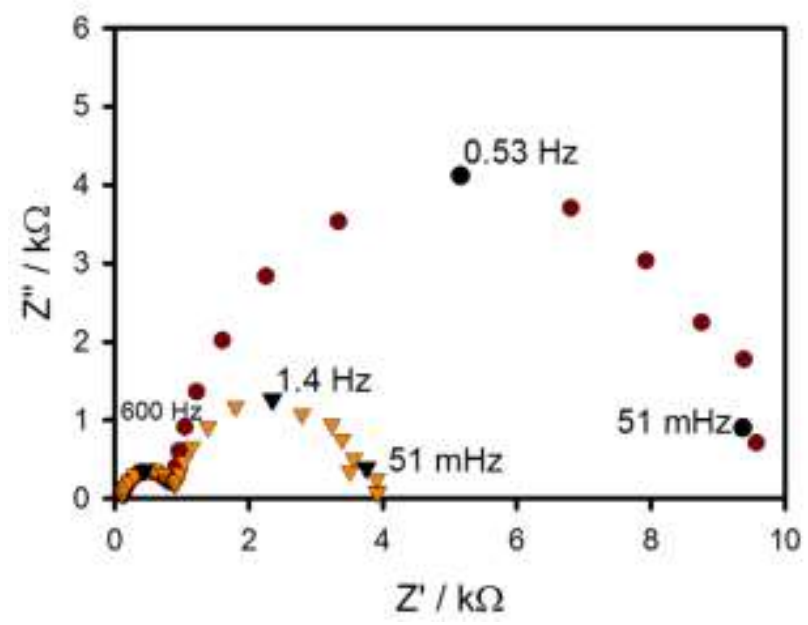

Figure 5. Nyquist plots for IS data measured at $\mathrm{pH} 6.9$ at $0.65 \mathrm{~V}$ vs $\mathrm{Ag} / \mathrm{AgCl}$ (red circles) and $0.70 \mathrm{~V}$ vs $\mathrm{Ag} / \mathrm{AgCl}$ (orange triangles) under 1 sun illumination. Several frequencies (black symbols) are labeled. 
From the outset we wish to show the close relationship between the $J(V)$ response and the impedance results. Therefore, Figure 6 shows a plot of the total resistance, $R_{\text {tot }}$ ( $R_{\text {tot }}=R_{S}+R_{\text {trapping }}+R_{c t \text { trap }}$ ), directly measured by IS compared to that obtained by the derivation of $R_{t o t}$ from the $J(V)$ curves as in Eq. (1). It is observed that both quantities are coincident, within experimental error. There is thus a perfect correlation between the total resistance, $R_{t o t}$, as a function of potential derived from the $J(V)$ curves (lines) and that from the fitted IS data (symbols) for different light intensities. The excellent agreement between the $R_{\text {tot }}$ calculated from the $J(V)$ curve and the IS parameters constitutes a strong evidence of the validity of our approach.

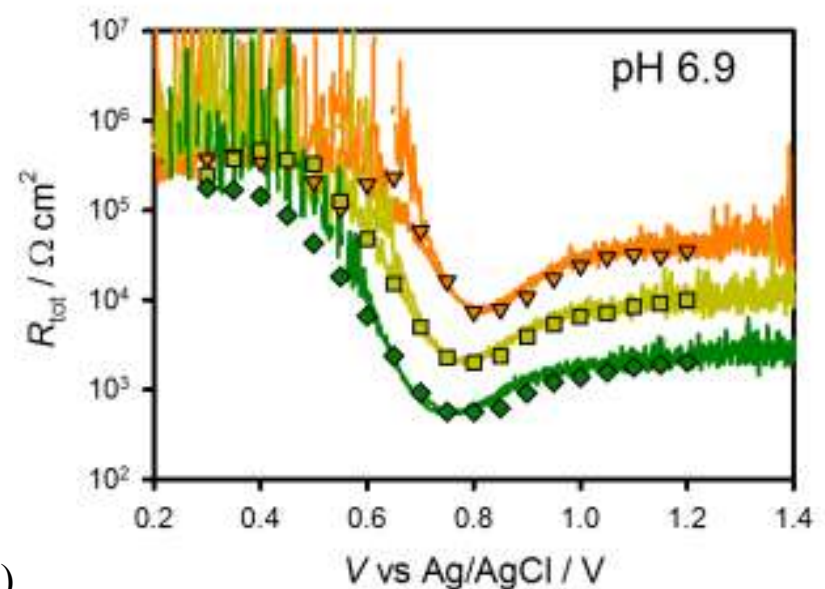

a)

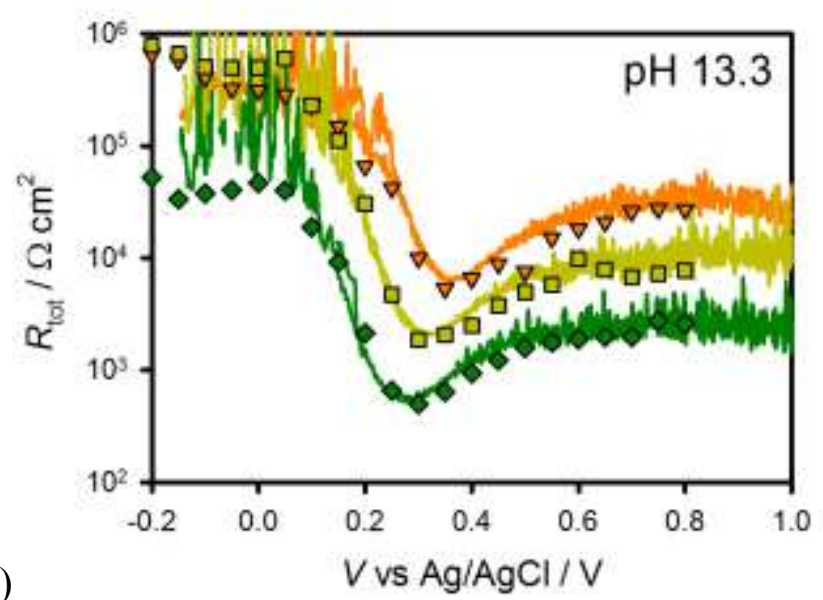

Figure 6. $\quad R_{\text {tot }}$ values determined by calculating $\mathrm{d} V / \mathrm{d} J$ from the $J(V)$ curves in figure 4 (lines) as well as the calculated $R_{\text {tot }}$ values by adding the resistances associated with charge transfer from IS (symbols) in pH 6.9 (a) and pH 13.3 (b) electrolytes. The colors correspond to different light intensities: 0.1 sun (orange triangles), 0.33 sun (yellow squares), and 1 sun (green diamonds). 
The values for the parameters extracted from fitting the IS data at $\mathrm{pH} 6.9$ and $\mathrm{pH} 13.3$ ( $C_{\text {bulk }}, R_{\text {trapping }}, C_{\text {trap }}$ and $R_{\text {ct,trap }}$ ) at the different light intensities are shown in Figure 7(a) and 7(b). The series resistance, $R_{s}$, was essentially constant and small, which is consistent with ohmic behavior at the FTO/hematite interface $46-48$ We note that the increase of $C_{\text {trap }}$ is correlated to the decrease of $R_{c t \text { trap }}$, it being the case that both $C_{\text {trap }}$ and $R_{c t \text { trap }}$ shift in concert for the different illumination conditions tested. This clearly indicates that hole transfer for the water splitting reaction takes place through the surface state. We note that similar behavior was obtained for multiple photoelectrodes, including those prepared with different thicknesses. Moreover, the photocurrent onset is also coincident with the charging of this surface state and the decrease of $R_{c t, t r a p}$, further confirming that charge transfer takes place from this surface state (Fig. 8). In contrast, the value of $R_{c t}$ in the dark is large and essentially constant indicating slow water oxidation kinetics from valence band holes. Obviously, photoexcitation of hematite is required to supply holes to the surface state, hence the dark characteristics are very different from those under illumination.

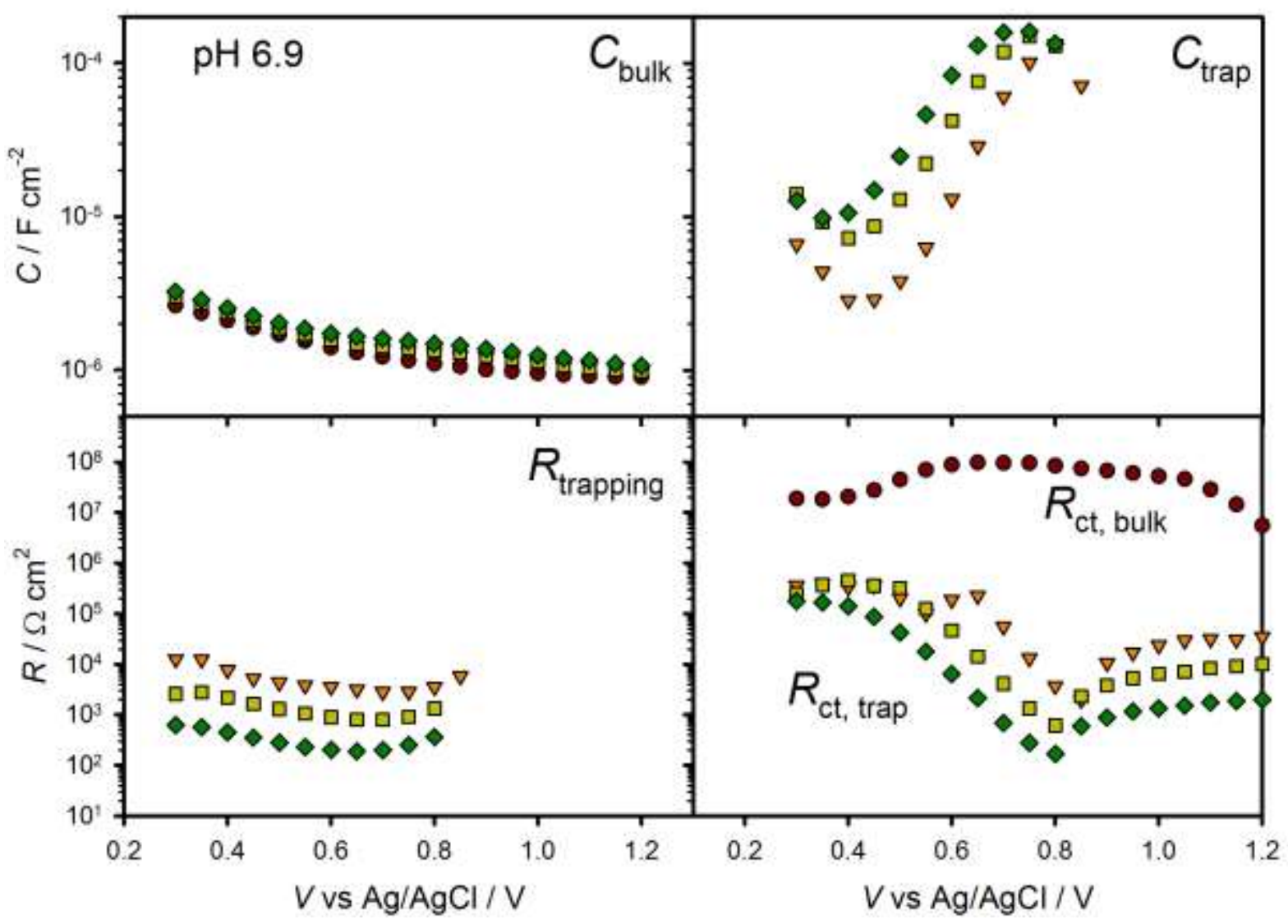

a) 


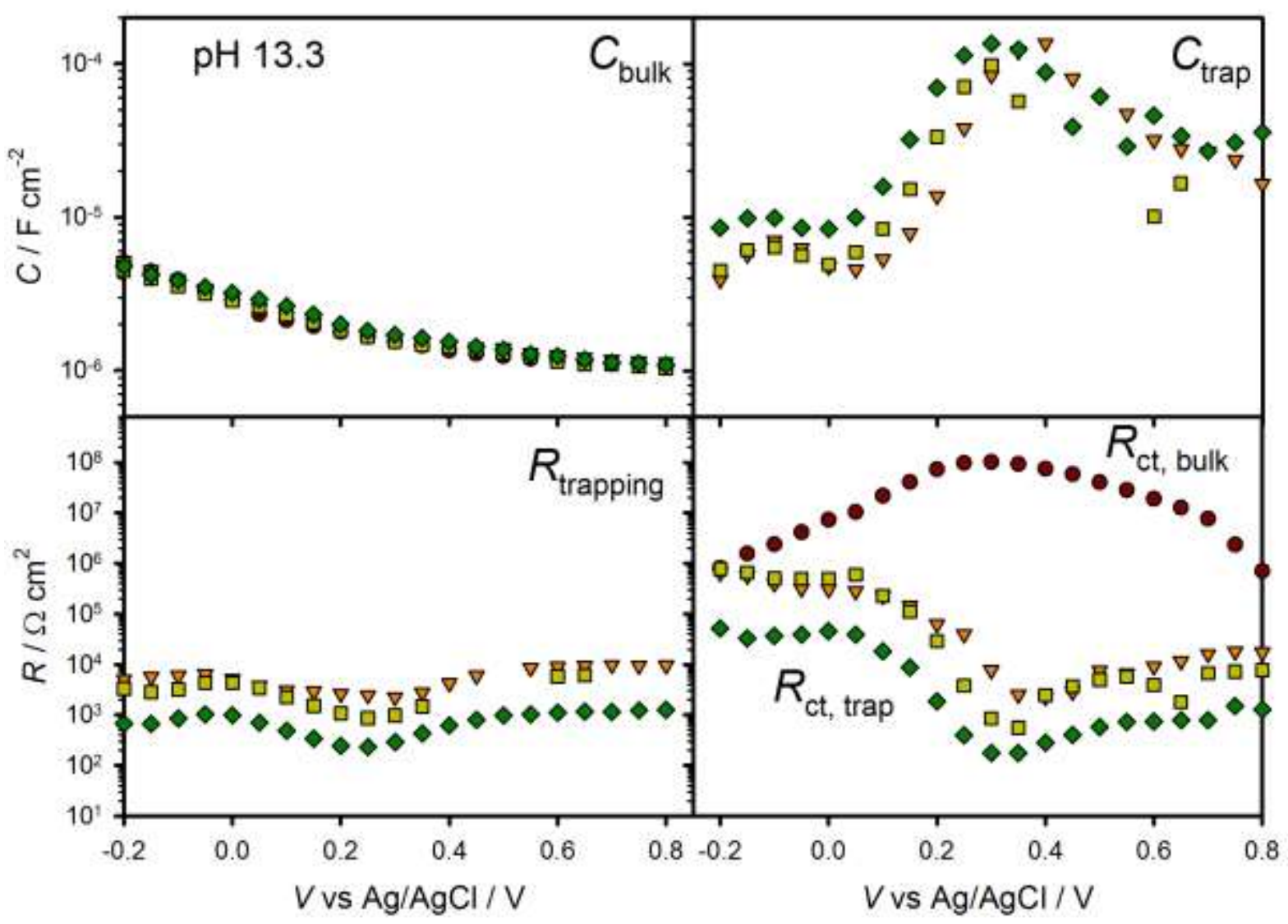

b)

Figure 7. Plots of equivalent circuit parameters obtained from fitting IS data in the dark (red circles), at 0.1 sun (orange triangles), 0.33 sun (yellow squares), and 1 sun (green diamonds) light intensities (a) at $\mathrm{pH} 6.9$ and (b) at $\mathrm{pH} 13.3$.

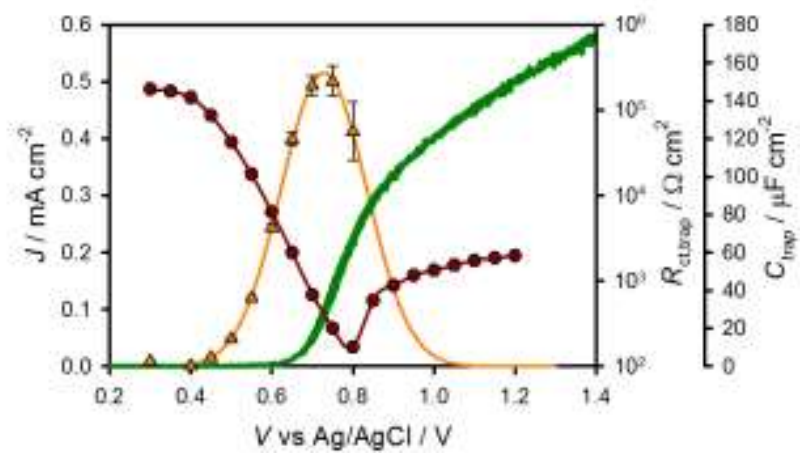

Figure 8. $J(V)$ curve (green solid line), $C_{\text {trap }}$ (orange triangles) and $R_{c t \text { trap }}$ (red circles) values obtained for a $60 \mathrm{~nm}$ hematite electrode under 1 sun illumination and $\mathrm{pH} 6.9$.

The values for $C_{\text {trap }}$ displayed in Figure 7 show Gaussian behavior when the sample is 
illuminated, in good agreement with the existence of a trap state described by equation 3 . The peak of $C_{\text {trap }}$ shifts to more negative potentials with increasing light intensity at $\mathrm{pH}$ 6.9. At $\mathrm{pH}$ 13 , however, there is no clear trend in the $C_{\text {trap }}$ peak with light intensity. The total magnitude of $C_{\text {trap }}$ increases with light intensity at $\mathrm{pH} 6.9$, however at $\mathrm{pH} 13.3$ the magnitude is lower compared to $\mathrm{pH} 6.9$ and essentially constant for the different illumination intensities. Comparing the position of the $C_{\text {trap }}$ peaks at both $\mathrm{pH}$ values, there is a shift by approximately $59 \mathrm{mV} / \mathrm{pH}$ unit, demonstrating Nernstian behavior. The $C_{\text {trap }}$ values can be used to calculate the energetic distribution of the density of states, $g\left(E_{F n}\right)$ or DOS, by 294

$$
C_{\text {trap }}(E)=q g\left(E_{F n}\right)
$$

The calculated DOS for hematite at $\mathrm{pH} 6.9$ and 13.3 can be seen in Figure 9(a) plotted vs $\mathrm{Ag} / \mathrm{AgCl}$ under different light intensities. A plot of the DOS vs RHE under 1 sun is also shown in Figure 9(b). The formal potential for water oxidation (1.23 V vs RHE) is also included. It is worth noting that the energetic distribution of the density of trap states peaks are very close to the formal potential of the oxygen evolution reaction, particularly at $\mathrm{pH}$ 13.3. This result suggests an equilibration of trap state energy and hole accepting species in the electrolyte. Since the water oxidation reaction involves the participation of four holes 50 some complications in interpretation arise from the fact that holes have to be stored in intermediate states 11

The details of the mechanism of water oxidation with metal oxide electrodes are still not clear, however it is generally believed to proceed from one or more surface hydroxyl (M$\mathrm{OH}_{\mathrm{x}}$ ) intermediate states formed from hole transfer to a surface coordinated water and a concomitant deprotonation step $51-54$ We therefore suggest that the surface state capacitance that builds up during water photo-oxidation is due to a $\mathrm{M}-\mathrm{OH}_{\mathrm{x}}$ intermediate. It is well known that metal oxide electrodes in contact with aqueous solutions have hydroxy-terminated surfaces; it is the protonation/deprotonation equilibrium of these $\mathrm{M}-\mathrm{OH}_{\mathrm{x}}$ sites which gives rise to the $\mathrm{pH}$-dependent variation of the band energies of metal oxide electrodes $4 \|_{5}^{5}$ in good agreement with the Nernstian behavior of the photoelectrodes observed in the present study. This could additionally account for the somewhat different IS behavior observed for water oxidation in $\mathrm{pH} 6.9$ and 13.3 electrolytes since the surface termination chemistry would be different. More work is needed to clarify these points, however, which is the subject of ongoing research in our labs. Nevertheless, this demonstrates the utility of employing IS in combination with $J(V)$ measurements in understanding water splitting reactions. 


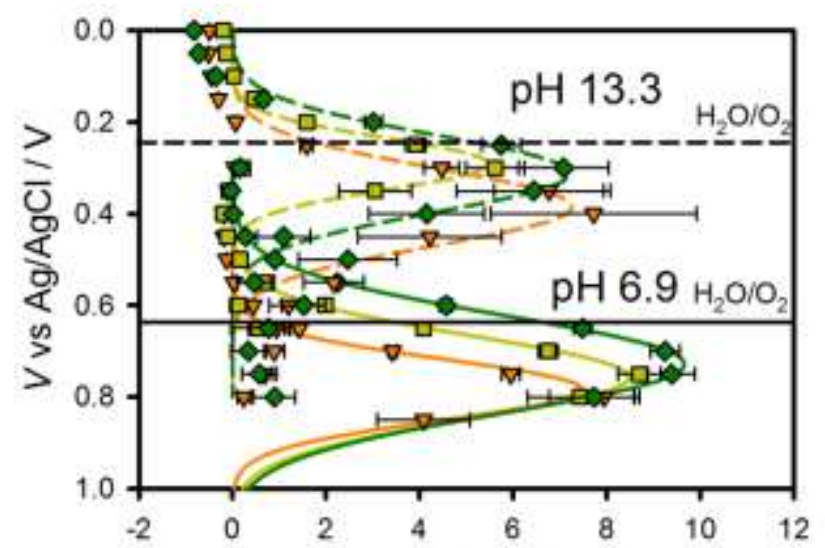

a)

DOS $/ 10^{14} \mathrm{~cm}^{-3} \mathrm{eV}^{-1}$

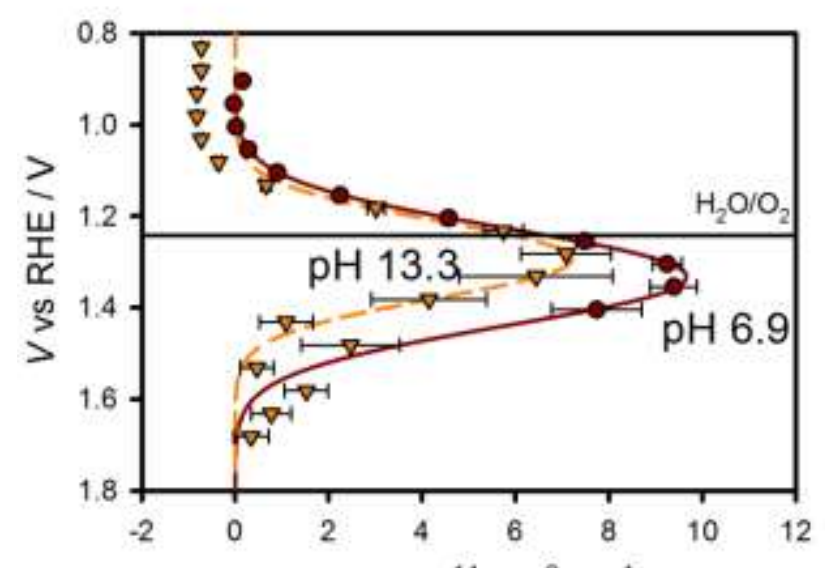

b)

DOS $/ 10^{14} \mathrm{~cm}^{-2} \mathrm{eV}^{-1}$

Figure 9. a) DOS as a function of potential for $\mathrm{pH} 6.9$ (solid lines) and $\mathrm{pH} 13.3$ (dashed lines) for 0.1 sun (orange triangles), 0.33 sun (yellow squares), and 1 sun (green diamonds) light intensities. b) DOS curves under 1 sun illumination vs. the RHE reference.

The values for $C_{\text {bulk }}$ do not show a significant trend in the logarithmic representation of Figure 6. These values were fit using the Mott Schottky equation (eq. 3). The resultant Mott-Schottky plots are displayed in Figures 10(a) for $\mathrm{pH} 6.9$ and 10(b) for $\mathrm{pH}$ 13.3. The calculation of the flatband potential, $E_{f b}$, and the dopant density, $N_{D}$, for different light intensities, can be seen in Table 1 . The dopant density, $N_{D}$ is practically constant for all the tested conditions, in the range $3 \times 10^{18}-6 \times 10^{18} \mathrm{~cm}^{-3}$ in good correspondence with previous values reported for this material ${ }^{40}$ The identity of these dopants has not been confirmed, due to the very small amount of impurity needed to produce these modest doping levels in the thin films studied. The typical assignment of oxygen vacancies for metal oxide electrodes, which can be related to the annealing and cooling of iron oxide independent of preparation method, may be applicable $4{ }_{40157}$ A recent report on the mechanism of the ALD of hematite suggests 
the dopants are due to a trace $\mathrm{Nb}$ impurity in the ALD reactor; thus the n-type doping may be due to $\mathrm{Nb}$ or other residual metal contamination in our ALD reactor ${ }^{58}$ In any case, we consistently obtain these doping levels, which is typical of hematite and other metal oxide electrodes 4 At 48 At $\mathrm{pH}$ 6.9, there is an obvious positive shift in the Mott Schottky plot under illumination; the shift increases with light intensity. This behavior is consistent with surface state charging as described by Memming et. al 20 In quantitative terms, the shift of the flat band potential $\left(\Delta E_{f b}\right)$ can be related to that derived from the charging of the surface states, which is calculated from $\Delta V_{\text {charging }}=Q_{\text {tot }} / C_{H}$. The total charge $Q_{\text {tot }}$ is obtained by integration of $C_{\text {trap }}$ with voltage as:

$$
Q_{\text {tot }}=\int C_{\text {trap }} d V
$$

In the supplementary information (Table SI1), we list the values $Q_{t o t}$ for the different $\mathrm{pH}$ and illumination intensities tested. While there is good qualitative agreement between $\Delta E_{f b}$ and $\Delta V_{\text {charging }}$, good quantitative agreement is obtained only when the value of Helmholtz capacitance is $C_{H}=2 \times 10^{-4} \mathrm{~F} \mathrm{~cm}^{-2}$ (Table 2). We note that this is an extremely high value for a Helmholtz capacitance.

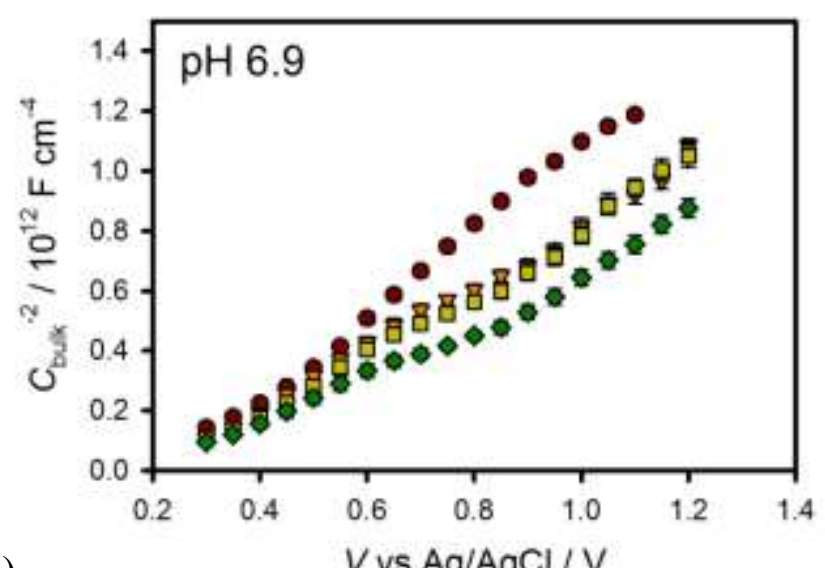

a) 


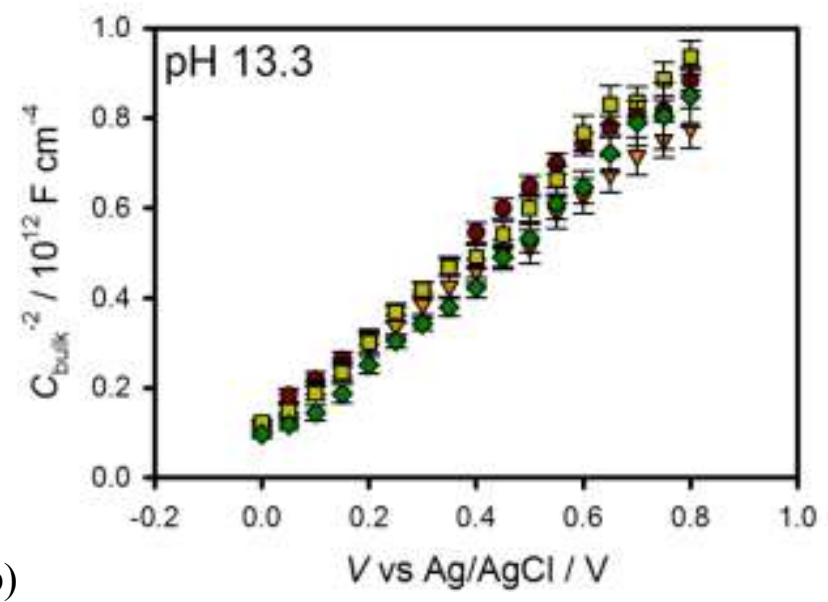

b)

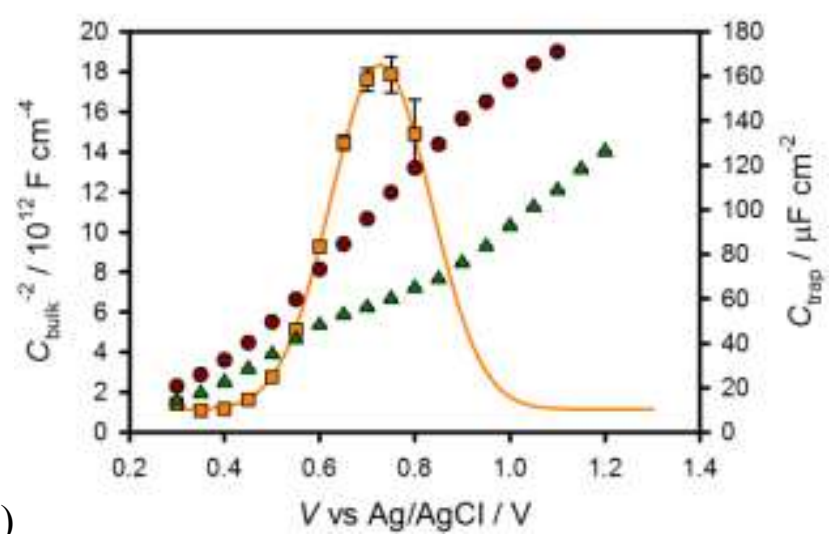

Figure 10. a) Mott Schottky plot at pH 6.9 under 0.1 sun (orange triangles), 0.33 sun (yellow squares), and 1 sun (green diamonds) light intensities and in the dark (red circles). b) Mott Schottky plot at pH 13.3 under 0.1 sun (orange triangles), 0.33 sun (yellow squares), and 1 sun (green diamonds) light intensities and in the dark (red circles). c) Mott Schottky plots at pH 6.9 in the dark (red circles) and under 1 sun (green diamonds). A plot of the trap state capacitance, $C_{\text {trap }}$, (orange squares) is superimposed to show the Fermi level pinning.

There is also a flat region in the Mott Schottky plot under illumination from approximately $0.6 \mathrm{~V}$ to $0.9 \mathrm{~V}$ vs $\mathrm{Ag} / \mathrm{AgCl}$ at $\mathrm{pH}$ 6.9. This flat region corresponds to the peak of the $C_{\text {trap }}$ under illumination. Figure 10(c) shows a plot of $C_{\text {trap }}$ superimposed on the Mott Schottky plots at pH 6.9. This is a clear example of Fermi level pinning. At pH 13, the magnitude of the surface state capacitance is lower compared to $\mathrm{pH} 6.9$ (Fig. 6). Consequently, a lower positive shift of the flat band potential can be anticipated by charging the surface state. This is indeed observed in Figure 10(b). The quantitative correlation between $\Delta E_{f b}$ and $\Delta V_{\text {charging }}$ is also shown in Table 2. There is good agreement except for the value obtained at 0.1 sun illumination. Again, the required values of $C_{H}$ to produce good quantitative agreement are too high for a double layer capacitance and further research is 
needed to understand this behavior.

\section{Conclusions}

Impedance Spectroscopy was employed to investigate the photoelectrochemical behavior of hematite electrodes under water splitting conditions. The Impedance spectra are characterized by the existence of a prominent surface state, which follows classical behavior in terms of capacitive features and the dependence on voltage and illumination intensity 18 The strong correlation between the $C_{\text {trap }}$ peak with the $R_{c t \text { trap }}$ valley and the photocurrent onset as illustrated in Figure 8, together with the perfect correlation between $R_{\text {tot }}$ from both IS and derivation of the steady state $J(V)$ curve clearly indicates that the hole-transfer step leading to water oxidation takes place predominantly from surface trapped holes, and not directly from valence band holes. This result represents an important step in understanding the mechanism of water oxidation at metal oxide electrodes; it should also provide new insight into the effects of surface modification strategies previously reported 45 The charging of the surface state was also used to explain flat band potential shifts under illumination, however an unusually large value of the Helmholtz capacitance is required. Thus, in addition to providing new insight into the water oxidation process, a methodology is presented which will be of great utility in further investigations of different configurations of hematite electrodes in order to determine their charge collection efficiency and surface catalytic properties.

Table 1. Parameters derived from the Mott Schottky plots under illumination. Calculations assume $k=32$.

\begin{tabular}{|c|c|c|c|c|}
\hline & \multicolumn{2}{|c|}{$\mathrm{pH} 7$} & \multicolumn{2}{c|}{$\mathrm{pH} 13.2$} \\
\hline $\begin{array}{c}\text { Illumination } / \\
\text { Sun }\end{array}$ & $\begin{array}{c}E_{\mathrm{fb}} / \mathrm{V} \text { vs } \\
\mathrm{Ag} / \mathrm{AgCl}\end{array}$ & $\mathrm{N}_{\mathrm{D}} / \mathrm{cm}^{-3}$ & $\begin{array}{c}E_{\mathrm{fb}} / \mathrm{V} \mathrm{vs} \\
\mathrm{Ag} / \mathrm{AgCl}\end{array}$ & $\mathrm{N}_{\mathrm{D}} / \mathrm{cm}^{-3}$ \\
\hline $\begin{array}{c}0 \text { (Randle } \\
\text { circuit) }\end{array}$ & 0.25 & $3 \times 10^{18}$ & -0.22 & $4.9 \times 10^{18}$ \\
\hline 0.1 & 0.33 & $3.6 \times 10^{18}$ & -0.20 & $5.8 \times 10^{18}$ \\
\hline 0.33 & 0.38 & $3.4 \times 10^{18}$ & -0.13 & $4.7 \times 10^{18}$ \\
\hline 1 & 0.45 & $3.8 \times 10^{18}$ & -0.07 & $4.8 \times 10^{18}$ \\
\hline
\end{tabular}

Table 2. Correlation of the voltage shift in flat-band potential $\Delta V_{f b}$ and that calculated 
from the charging of the surface state $\Delta \mathrm{V}_{\text {charging }}$ (with $C_{\mathrm{H}}=2 \times 10^{-4} \mathrm{~F} \cdot \mathrm{cm}^{-2}$ ).

\begin{tabular}{|c|c|c|c|c|}
\hline & \multicolumn{2}{|c|}{$\mathrm{pH}$} & \multicolumn{2}{c|}{$\mathrm{pH} 13$} \\
\hline & $\Delta \mathrm{V}_{\text {charging }}(\mathrm{mV})$ & $\Delta \mathrm{V}_{\mathrm{FB}}(\mathrm{mV})$ & $\Delta \mathrm{V}_{\text {charging }}(\mathrm{mV})$ & $\Delta \mathrm{V}_{\mathrm{FB}}(\mathrm{mV})$ \\
\hline 0.1 sun & 98 & 76 & 121 & 14 \\
\hline 0.33 sun & 153 & 130 & 61.4 & 82 \\
\hline 1 sun & 204 & 197 & 121 & 147 \\
\hline
\end{tabular}

\section{Acknowledgements}

TWH acknowledges the Donors of the American Chemical Society Petroleum Research Fund (\#51099-DNI10) for support of this research. JB acknowledges support by projects from Ministerio de Ciencia e Innovación (MICINN) of Spain (Consolider HOPE CSD200700007), and Generalitat Valenciana (PROMETEO/2009/058). SG acknowledges support by MICINN of Spain under the Ramon y Cajal programme.

\section{Supporting I nformation}

The parameters determined when fitting IS data to an alternative model shown in figure 1c, and the justification of using the model shown in figure 1d can be found in supporting information. Also included is a table with the calculated charge stored in the surface trap states. This material is available free of charge via the internet at http://pubs.acs.org

\section{References}

(1) Walter, M. G.; Warren, E. L.; McKone, J. R.; Boettcher, S. W.; Mi, Q. X.; Santori, E. A.; Lewis, N. S. Chemical Reviews 2010, 110, 6446.

(2) Bolts, J.; Wrighton, M. The Journal of Physical Chemistry 1976, 80, 2641.

(3) Butler, M. A.; Ginley, D. S. Journal of Material Science 1980, 15, 1.

(4) Lin, Y.; Yuan, G.; Sheehan, S.; Zhou, S.; Wang, D. Energy \& Environmental Science 2011.

(5) John, H. K.; Karl W. Frese, Jr. Journal of The Electrochemical Society 1978, $125,709$.

(6) Murphy, A. B.; Barnes, P. R. F.; Randeniya, L. K.; Plumb, I. C.; Grey, I. E.; Horne, M. D.; Glasscock, J. A. International Journal of Hydrogen Energy 2006, 31, 1999.

(7) Kay, A.; Cesar, I.; Grätzel, M. Journal of the American Chemical Society 2006, 128, 15714. 
(8) Sivula, K.; Zboril, R.; Le Formal, F.; Robert, R.; Weidenkaff, A.; Tucek, J.; Frydrych, J.; Grätzel, M. Journal of the American Chemical Society 2010, 132, 7436.

(9) Barroso, M.; Cowan, A. J.; Pendlebury, S. R.; Gral’tzel, M.; Klug, D. R.; Durrant, J. R. Journal of the American Chemical Society 2011, 133, 14868.

(10) Pendlebury, S. R.; Barroso, M.; Cowan, A. J.; Sivula, K.; Tang, J.; Gratzel, M.; Klug, D.; Durrant, J. R. Chemical Communications 2011, 47, 716.

(11) Wijayantha, K. G. U.; Saremi-Yarahmadi, S.; Peter, L. M. Physical Chemistry Chemical Physics 2011, 13, 5264.

(12) Dotan, H.; Sivula, K.; Gratzel, M.; Rothschild, A.; Warren, S. C. Energy Environ. Sci. 2010.

(13) George, S. M. Chem. Rev. 2010, 110, 111.

(14) Hamann, T. W.; Martinson, A. B. F.; Elam, J. W.; Pellin, M. J.; Hupp, J. T. Adv. Mater. 2008, 20, 1560.

(15) Hamann, T. W.; Martinson, A. B. F.; Elam, J. W.; Pellin, M. J.; Hupp, J. T. J. Phys. Chem. C2008, 112, 10303.

(16) Lin, Y. J.; Zhou, S.; Sheehan, S. W.; Wang, D. W. J. Am. Chem. Soc. 2011, 133, 2398.

(17) Frese, K. W.; Morrison, S. R. Journal of the Electrochemical Society 1979, $126,1235$.

(18) Allongue, P.; Cachet, H. Journal of the Electrochemical Society 1985, 132, 45.

(19) Lincot, D.; Vedel, J. Journal of Electroanalytical Chemistry and Interfacial Electrochemistry 1987, 220, 179.

(20) Kelly, J. J.; Memming, R. Journal of the Electrochemical Society 1982, 192, 730.

(21) Reichman, J. Applied Physics Letters 1980, 36, 574.

(22) Hens, Z. Journal of Physical Chemistry B 1999, 103, 122.

(23) Leng, W. H.; Zhang, Z.; Zhang, J. Q.; Cao, C. N. The Journal of Physical Chemistry B 2005, 109, 15008.

(24) Ponomarev, E. A.; Peter, L. M. Journal of Electroanalytical Chemistry 1995, 397,45 .

(25) Vanmaekelbergh, D.; Cardon, F. Journal of Physics D-Applied Physics 1986, $19,643$.

(26) Bisquert, J. Journal of Electroanalytical Chemistry 2010, 646, 43.

(27) Vanmaekelbergh, D.; Cardon, F. Journal of Physics D: Applied Physics 1986, $19,643$.

(28) Glasscock, J. A.; Barnes, P. R. F.; Plumb, I. C.; Bendavid, A.; Martin, P. J. Thin Solid Films 2008, 516, 1716.

(29) Bisquert, J. Phys. Chem. Chem. Phys. 2003, 5, 5360. 
(30) Bisquert, J. Physical Chemistry Chemical Physics 2008, 10, 49.

(31) Hoffmann, P. M.; Oskam, G.; Searson, P. C. Journal of Applied Physics 1998, 83, 4309.

(32) Scurtu, R.; Ionescu, N. I.; Lazarescu, M.; Lazarescu, V. Physical Chemistry Chemical Physics 2009, 11, 1765.

(33) Gärtner, W. Physical Review 1959, 116, 84.

(34) Salvador, P. Journal of Physical Chemistry B 2001, 105, 6128.

(35) Vanmaekelbergh, D.; Gomes, W. P.; Cardon, F. Journal of the Electrochemical Society 1987, 134, 891.

(36) Bisquert, J.; Fabregat-Santiago, F.; Mora-Sero, I.; Garcia-Belmonte, G.; Barea, E. M.; Palomares, E. Inorganica Chimica Acta 2008, 361, 684.

(37) Bisquert, J.; Vikhrenko, V. S. Journal of Physical Chemistry B 2004, 108, 2313.

(38) Mora-Sero, I.; Bisquert, J. Nano Letters 2003, 3, 945.

(39) Simmons, J. G.; Taylor, G. W. Physical Review B-Solid State 1971, 4, 502.

(40) Klahr, B. M.; Martinson, A. B. F.; Hamann, T. W. Langmuir 2011, 27, 461.

(41) Libera, J. A.; Hryn, J. N.; Elam, J. W. Chemistry of Materials 2011, 23, 2150.

(42) Anderman, M.; Kennedy, J. H. Journal of the Electrochemical Society 1984, $131,1565$.

(43) Klahr, B. M.; Hamann, T. W. Journal of Physical Chemistry C 2011, 115, 8393.

(44) Launay, J. C.; Horowitz, G. Journal of Crystal Growth 1982, 57, 118.

(45) Le Formal, F.; Tetreault, N.; Cornuz, M.; Moehl, T.; Gratzel, M.; Sivula, K. Chemical Science 2011, 2, 737.

(46) Glasscock, J. A.; Barnes, P. R. F.; Plumb, I. C.; Savvides, N. Journal of Physical Chemistry C 2007, 111, 16477.

(47) Morrison, S. R. Electrochemistry at Semiconductor and Oxidized Metal Electrodes, Plenum: New York, 1980.

(48) Finklea, H. O. Semiconductor Electrodes, Elsevier: Amsterdam, 1988.

(49) Fabregat-Santiago, F.; Garcia-Belmonte, G.; Mora-Sero, I.; Bisquert, J. Physical Chemistry Chemical Physics 2011, 13, 9083.

(50) Tang, J. W.; Durrant, J. R.; Klug, D. R. Journal of the American Chemical Society 2008, 130, 13885.

(51) Cook, T. R.; Dogutan, D. K.; Reece, S. Y.; Surendranath, Y.; Teets, T. S.; Nocera, D. G. Chemical Reviews 2010, 110, 6474.

(52) Conway, B. E.; Tilak, B. V. Advances in Catalysis 1992, 38, 1.

(53) Montoya, J. F.; Peral, J.; Salvador, P. Chemphyschem 2011, 12, 901.

(54) Salvador, P. Journal of the Electrochemical Society 1981, 128, 1895. 
(55) Hamann, T. W.; Lewis, N. S. Journal of Physical Chemistry B 2006, 110, 22291.

(56) Gardner, R. F. G.; Sweett, F.; Tanner, D. W. Journal of Physics and Chemistry of Solids 1963, 24, 1183.

(57) Gardner, R. F. G.; Tanner, D. W.; Sweett, F. Journal of Physics and Chemistry of Solids 1963, 24, 1183.

(58) Martinson, A. B. F.; DeVries, M. J.; Libera, J. A.; Christensen, S. T.; Hupp, J. T.; Pellin, M. J.; Elam, J. W. The Journal of Physical Chemistry C 2011, 115, 4333. 
TOC graphic

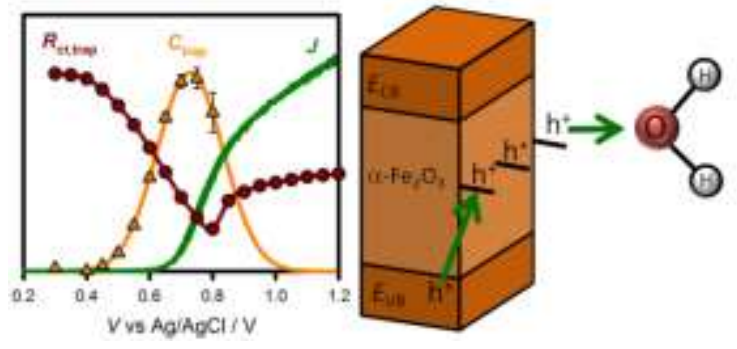

